\title{
Diacronie
}

Studi di Storia Contemporanea

$N^{\circ} 18,2$ | 2014

Le esposizioni: propaganda e costruzione identitaria

\section{Exposition universelle de 1878. L'archéologie au service des contestations contemporaines}

\section{Bastien Noël}

\section{(2) OpenEdition}

\section{Journals}

\section{Édition électronique}

URL : http://journals.openedition.org/diacronie/1478

DOI : 10.4000/diacronie. 1478

ISSN : 2038-0925

Éditeur

Association culturelle Diacronie

\section{Référence électronique}

Bastien Noël, «Exposition universelle de 1878. L'archéologie au service des contestations contemporaines », Diacronie [En ligne], N 18, 2 | 2014, document 11, mis en ligne le 01 juin 2014, consulté le 01 mai 2019. URL : http://journals.openedition.org/diacronie/1478; DOI : 10.4000/ diacronie. 1478 


\section{Diacronie}

\section{1/}

\section{Exposition universelle de 1878. L'archéologie au service des contestations contemporaines}

Bastien NOËL*

L'Exposition universelle de 1878 prend place entre le Champ de Mars et le Trocadéro. Après la défaite de Sedan et la Commune, l'événement offre au monde l'image du redressement français et affirme la IIIe République. Malgré leur caractère commercial, les expositions universelles ne se limitent pas à une célébration des créations modernes. Présentés au sein de rétrospectives, les œuvres archéologiques de l'Exposition de 1878 imprègnent sensiblement leur époque. Les dépôts antiques éveillent les nationalismes et sous-tendent des contestations politiques ou religieuses. Investis de ces discours, ils soulignent par contraste le progrès humain, ou soutiennent par comparaison, la continuité de certaines pratiques industrielles.

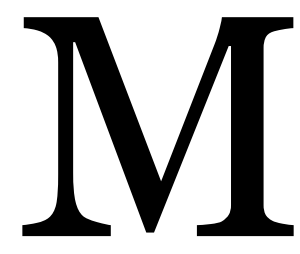

algré leur caractère commercial, les expositions universelles ne se limitent pas à une célébration de l’industrie. Au sein de ces concours internationaux, l'ancienneté des cultures fait l'objet d'expositions

rétrospectives. Présentés aux côtés des produits les plus innovants, les dépôts antiques de l'Exposition de 1878 soulignent, par contraste, le progrès humain ou soutiennent, par comparaison, la continuité de certaines pratiques. Cette confrontation entre passé et présent nourrit les idéologies de la fin du siècle. Ainsi, deux leviers sont sollicités pour instrumentaliser les objets archéologiques à l'Exposition universelle. Le premier est celui de l'éloignement temporel: l'histoire humaine telle que la redéfinit la science archéologique met à défaut le traditionalisme religieux. Via le mythe gaulois, 
elle porte aussi un discours nationaliste qui constitue l'enjeu véritable de toute Exposition universelle. Le second levier est celui de la continuité. Les objets antiques attestent de l'ancienneté de certaines pratiques et mettent en exergue le prestige d'une industrie en assurant son ancrage historique millénaire.

\section{L'argument du passé au secours des contestations contemporaines}

\subsection{Dénégation d'un traditionalisme religieux}

En France, l'Exposition universelle de 1878 s'ouvre dans une période riche en initiatives de laïcisation ${ }^{1}$. Les progressistes profitent de l'exposition de vestiges archéologiques pour mettre en doute l'histoire chrétienne et initier un mouvement qui aboutit à plusieurs mesures dès l'année suivante ${ }^{2}$.

Dans une critique formulée à l'égard du «Darwinisme »soutenu au Pavillon de l'anthropologie3 du quai de Billy, Henry des Houx, oppose le secrétaire Paul Topinard4, «avocat des singes »et responsable de la vitrine 23 consacrée aux rapprochements entre l'homme et le primate, à Armand de Quatrefages 5 , qui est de ceux qui ont "protesté contre la témérité des hypothèses darwinistes ». La confrontation entre le président de la commission et son secrétaire Paul Topinard transpose ce conflit idéologique au sein même du comité organisateur de l'exposition anthropologique et révèle la complexité du débat religieux contemporain. On ne peut s'en tenir à une opposition entre une classe scientifique darwinienne et un ensemble de fidèles aux mœurs conservatrices.

Les anthropologues ne sont d'ailleurs pas les seuls acteurs de cette contestation. Plusieurs commentateurs de l'exposition de l'art ancien, présentée au sein du Palais du

\footnotetext{
${ }^{1}$ Dès 1871, la Commune anticipe la séparation de l'Église et de l'État ; en 1875, le député Alfred Naquet propose de rétablir le divorce pour faute de la loi de 1792. Les deux initiatives se soldent par un échec.

${ }^{2}$ L'année 1879 marque en effet un tournant décisif: l'arrivée à la présidence de la République de Jules Grévy et la nomination de Jules Ferry au poste de ministre de l'Instruction publique ébranlent l'intransigeance de l'Église catholique.

3 La Défense, 10 août 1878. Archives nationales de France F/12/3296: Commissariat général/Services de l'État/Direction de la presse/ministère de l'intérieur. Août-décembre 1878. L'auteur doute de la crédibilité scientifique de cette thèse et en appelle à ce qu'il considère vraisemblablement comme du bon sens: «Est-il vraiment bien moral et bien spirituel de démontrer la parenté de M. de Lesseps avec un orang-outan, en passant par Troppmann ? Estce là un beau spectacle d'exposition, bien propre et la science française aux yeux des nations rivales?».

4 Médecin et anthropologue français (1830-1911). Secrétaire général de la commission d'initiative de l'exposition anthropologique, organisée dans le cadre de l'Exposition universelle de 1878.

5 Anthropologue français (1810-1892), il occupe en 1878 le poste de président de la commission d'initiative de l'exposition anthropologique.
} 
Trocadéro, appuient une pensée laïque sur leurs descriptions d'œuvres antiques. Dans L'art et l'industrie de tous les peuples à l'Exposition universelle, Henry Havard s'interroge sur l'ancienneté de l'homme et relève la cohabitation délicate entre le discours religieux et l'avancée de la recherche archéologique:

Voilà qu'aujourd'hui deux abbés, MM. Bourgeois et Delaunay, au mépris de la chronologie catholique, ont cru trouver des traces de l'industrie humaine dans les terrains pliocènes. Où s'arrêtera-t-on ${ }^{6}$ ?

À propos du moulage du buste en serpentine du roi Chefreu, l'auteur cite Auguste Mariette $^{7}$ et date l'objet d' «il y a six mille ans», avant d'ajouter: "c'est-à-dire précisément à l'époque où, suivant les dires de la Genèse, le Seigneur était occupé à la création du monde» ${ }^{8}$. La comparaison entre ce chef-d'œuvre statuaire et les productions contemporaines mises au jour en Europe occidentale révèle, selon-lui, l'obsolescence du discours religieux:

Embrassons d'un coup d'œil l'effroyable distance qui sépare la barbarie où croupissaient ces troglodytes dont nous parlions précédemment, de la civilisation grandiose que nous révèle cette admirable statue ; et dites-moi, je vous prie, ce que devient cette chronologie hiératique que nos enfants apprennent dans l'Histoire sainte et qu'on leur enseigne comme un acte de foi 9 .

La démonstration se poursuit à partir du décor de la tombe de Ti à Saqqarah:

Que leur manque-t-il donc à ces Égyptiens antiques ? [...] Les voilà donc complets, presque nos égaux, éprouvant les mêmes besoins que nous, goûtant les mêmes plaisirs, cultivant les mêmes vices. [...] Voyez [...] l'abîme effroyable qui sépare leur civilisation raffinée des grossiers vestiges de l'âge de pierre. Est-ce que tout ce traditionnel échafaudage de contes à dormir debout, dont on berce l'esprit de nos enfants, ne s'écroule pas devant une pareille démonstration ? Que reste-t-il de cette théorie du monde fabriqué en six jours ? Et continuera-t-on d'enseigner ce tissu de fables ineptes à des cerveaux incapables d'en contrôler les erreurs ${ }^{10}$ ?

\footnotetext{
${ }^{6}$ L'art et l'industrie de tous les peuples à l'Exposition Universelle de 1878. Description illustrée des merveilles du Champ de Mars et du Trocadéro par les écrivains spéciaux les plus autorisés. Paris, Librairie illustrée, 1879, p. 512.

7 Archéologue français. Chargé en 1867 puis en 1878 d'organiser la participation de l'Égypte à l'Exposition universelle de Paris.

${ }^{8}$ L'art et l'industrie de tous les peuples à l'Exposition Universelle de 1878, op.cit., p. 513.

9 Ibidem.

${ }^{10}$ Ibidem, p. 515 .
} 
Partant de l'ancienneté des civilisations, dont témoignent les œuvres égyptiennes exposées sous l'égide d'Auguste Mariette, Henry Havard met à défaut ce «labyrinthe de récits mensongers» ${ }^{11}$ que constituent les textes catholiques. Aussi les découvertes présentées au Trocadéro doivent-elles encourager le visiteur à' s'affranchir vis-à-vis des «ridicules entraves »et de l'«ignorance »auxquelles la tradition le condamne. «Il faut briser ces obstacles cependant, car c'est devant de pareilles difficultés que se sont arrêtées la plupart des civilisations stationnaires ${ }^{12}$ conclue l'auteur, en visionnaire de la loi de 1905 sur la séparation de l'Eglise et de l'Etat.

\subsection{Instrumentalisation politique et nationalisme français}

Valable au début du XIX ${ }^{\mathrm{e}}$ siècle, la conception d'un passé propre à la Gaule cède face aux recherches menées par les archéologues dans la première moitié du siècle. Dans toute l'Europe, les scientifiques échangent et communiquent l'avancée de leurs travaux. Congrès et colloques conduisent à l'élaboration d'une «histoire primitive de l'Europe» ${ }^{13}$ construite par l'apport de populations successives dont on trouve la trace dans plusieurs pays, dont la France.

Cependant, ces avancées ne sont pas immédiatement connues du grand public. L'histoire primant encore l'archéologie dans les mentalités contemporaines, la théorie d'un passé gaulois unique reste dominante dans les années 1870. Cette méconnaissance relève moins d'un désintérêt du public pour une science en construction que d'un obscurantisme entretenu par la classe savante. Sous son autorité, une habile sélection d'objets triés parmi les découvertes du siècle vient porter caution du «mythe gaulois» auprès du public. Cette instrumentalisation de l'archéologie se justifie par l'entreprise de redressement national initiée dans le dernier tiers du XIX ${ }^{\mathrm{e}}$ siècle. Après l'échec français de 1871, de nombreux intellectuels encouragent une quête identitaire dont Henri Martin ${ }^{14}$ se fait le pionnier. Ce dernier œuvre pour l'intégration des Gaulois à la mythologie républicaine et érige en exemple ces «ancêtres [qui] se sont battus et sacrifiés pour sauvegarder l’indépendance de leur territoire et l’intégrité de leur génie»15. Dans son Mémoire sur la Défense de Paris, Eugène Viollet-le-Duc ${ }^{16}$ soutient

\footnotetext{
${ }^{11}$ Ibidem.

${ }^{12}$ Ibidem, p. 516.

13 BERTRAND, Alexandre, Archéologie celtique et gauloise, Paris, Didier et C.ie, 1876, p. 402, cit. in GRAN-AYMERICH, Evelyne, Naissance de l'archéologie moderne: 1798-1945, Paris, Éditions du Centre National de la Recherche Scientifique (CNRS), 1998, p. 225.

14 Historien et homme politique français (1810-1883). Son étude contribue à l'essor des études celtiques et anthropologiques.

15 GRAN-AYMERICH, Evelyne, op. cit., p. 221.
} 
ce regard rétrospectif : «Nous ne devons pas étouffer, pour en rivaliser avec le germain, notre esprit Gaulois»17. Le passé glorieux de la France doit nourrir le redressement de la nation vaincue. À la gallomanie née sous Napoléon III succède après 1870 une étude qui se porte sur la succession des trois Gaule, celte, romaine et mérovingienne. Mais au lendemain de la défaite de Sedan, les deux dernières époques sont largement méprisées et les analogies se succèdent pour assimiler les Germains historiques aux ennemis Prussiens. L'admiration pour l'Allemagne savante a cessé. C'est vers une première France civilisée, antérieure aux invasions du Ve siècle que l'on se tourne. Le choix de la période satisfait aussi les désirs de laïcité contemporains en fondant les racines françaises non dans la foi chrétienne, mais dans une spiritualité magique et druidique. L’idéal gaulois primitif, affranchi du joug romain et des influences barbares est avancé comme modèle pour la IIIe République. Cette Gaule indépendante est «une grande République fédérative [...], où la femme marche l'égale de l'homme [...], [où] la liberté a vaincu la fatalité [...], [où] l'homme a sa conscience pour guide de ses actions» assure Eugène Bonnemère dans La Gaule en $1875^{18}$. On rappelle ailleurs le pouvoir militaire d'un empire qui «s'étendit de l'Espagne à l'Asie Mineure»19. Dans les dernières décennies du XIXe, «la Revanche et la République dictent le discours sur la Gaule»²0.

La cohabitation forcée entre les avancées anthropologiques et l'entretien d'un «passé gaulois» se reflète à l'Exposition universelle de 1878, au palais du Champ de Mars comme au Trocadéro. Dans l'enceinte du premier palais expose Évariste-Vital Luminais $^{21}$, artiste français «qui peint les anciens Gaulois comme s'il eût été élevé luimême chez les druides» ${ }^{22}$. De cette Gaule indépendante, l'exposition rétrospective du Trocadéro livre de nombreux vestiges. «Je parlais à l'instant des Gaulois, nos ancêtres ; on leur a fait une grande et large place au Trocadéro ${ }^{23}$ confirme Henry Havard. Portés par l'engouement, les commentateurs de l'exposition vont jusqu'à reconnaître des

\footnotetext{
${ }^{16}$ Historien de l'art (1814-1879), il devient la figure dominante de l'architecture française dans les années 1840-1870.

${ }_{17}$ Cit. in CARBONELL, Charles-Olivier, Après 1870: régénérescence de la France et naissance de la Gaule, in VIALLANEIX, Paul EHRARD (rec. par), Jean, Nos ancêtres, les Gaulois, Clermont-Ferrand, Universite de Clermont Ferrand II, 1982, p. 391.

${ }^{18}$ Cit. in CARBONELL, Charles-Olivier, op. cit., p. 393.

19 DURAND-LAÎNÉ, Adolphe, cit. in CARBONELL, Charles-Olivier, op. cit., p. 393.

${ }^{20}$ CARBONELL, Charles-Olivier, op. cit., p. 394.

${ }^{21}$ Peintre français (1821-1896), porteurs de l'idéologie de la IIIe République. Plusieurs de ses toiles sont présentés au Palais du Champ de Mars à l'Exposition universelle de 1878.

${ }_{22}$ BLANC, Charles, Les Beaux-Arts, in L'art et l'industrie de tous les peuples à l'Exposition universelle de 1878, cit., p. 164.

${ }_{23}^{23}$ L'art et l'industrie de tous les peuples à l'Exposition Universelle de 1878, cit., p. 511.
} 
objets «gaulois »dans chaque découverte issue du sol français et contenue dans un cadre chronologique débutant avec l'âge des métaux ${ }^{24}$.

À l'inverse, le Pavillon de l'anthropologie du quai de Billy semble coller plus étroitement aux considérations scientifiques de son temps. S’il consacre une partie de ses vitrines aux antiquités gauloises, l'appropriation nationaliste de ces objets y semble moins évidente. En effet, la position d'une histoire propre à la France est plus difficile à soutenir à mesure que les anthropologues remontent le fil de nos origines. Les dépôts du quai de Billy œuvrent davantage à une reconnaissance d'un passé européen commun qu'à la constitution d'un "passé gaulois ». Alors que le Palais du Trocadéro prend le parti d'isoler les sections étrangères dans son aile ouest, une confrontation fertile a pu s'établir à l'exposition anthropologique, où se trouvaient rassemblées dans un même espace les vestiges préhistoriques de plusieurs régions de l'Europe ${ }^{25}$.

Ainsi, l'instrumentalisation nationaliste des antiquités nationales à l'Exposition universelle de 1878 relève d'un phénomène complexe. En plein essor grâce au concours de scientifiques dans toute l'Europe, la discipline reste méconnue du grand public. Quand elle n'est pas simplement ignorée, l'archéologie est fréquemment assimilée à une science historique qui, par ses investigations, offre dans les années 1870 un modèle de développement pour le présent.

\section{Archéologie et continuité industrielle à l'Exposition universelle}

\subsection{Le rappel de la valeur industrielle des productions antiques}

L'Exposition universelle de 1878 témoigne d'une conception nouvelle de l'industrie. Dispersées dans des architectures pavillonnaires, les techniques sont concurrencées par la curiosité et le divertissement dont la place s'est considérablement accrue par rapport à la manifestation française de 1867. Ce nouveau rapport ne réside pas tant dans la disparition de la valeur du travail que dans sa dissolution au sein de présentations qui s'y rattachent moins directement. Parmi elles, les expositions archéologiques délivrent un discours qui assure une continuité entre les productions anciennes et l'industrie moderne. «Rien n'est plus intéressant, n'est plus instructif que la comparaison des trésors des arts et des industries du présent et du passé »lit-on dans la République

${ }^{24}$ MULLËR-SCHEESSEL, Nils, «Fair prehistory: archaeological exhibits at French Expositions universelles» in Antiquity, 75, 288, p. 400.

${ }_{25}$ Parmi lesquelles la France, le Royaume-Uni, l'Espagne, l'Italie, le Portugal, la Pologne, la Suède, la Norvège. 
française du 21 mai $1878^{26}$ à propos de la rétrospective de l'art ancien au Trocadéro. Si la dénomination a changé, cette présentation n'en reste pas moins l'héritière de la Galerie de l'histoire du Travail de l'Exposition universelle de 1867. Aussi le lien entre le monde antique et l'industrie contemporaine est-il étroit au Trocadéro. Dès 1877 , Gabriel de Mortillet ${ }^{27}$ associe les deux conceptions en annonçant que l'aile droite du palais présentera «le développement de l'art et de l'industrie, depuis les temps les plus reculés jusqu'à la Révolution française» ${ }^{28}$. Dans ce cadre, un lien est créé entre les objets antiques du Trocadéro et les technologies du palais du Champ de Mars : pour Henry Havard, les antiquités préhistoriques de l'aile ouest sont «le germe de ces formidables engins, de ces machines admirables qui grondent dans les halls gigantesques du grand palais de fer» ${ }^{29}$. Fréquemment évoquée ${ }^{30}$, cette dimension technique légitime la place occupée par une telle présentation dans une exposition industrielle et offre un nouveau regard sur ces objets du passé «longtemps méprisés, ignorés, dédaignés» ${ }^{1}$. Par leur nature fatalement artisanale, les œuvres anciennes de toutes origines répondent à cette ambition de faire cohabiter art et industrie. Le recours au terme de «produit "pour désigner les dépôts anciens accompagne ce rapprochement ${ }^{32}$. Le Rapport sur l'exposition de l'art ancien du Trocadéro se félicite ainsi d'offrir aux «arts et [...] procédés industriels de l'Égypte, à partir des époques les plus reculées, de l'Asie centrale de l'Extrême Orient, de la Grèce, de l'Italie [...], des représentants dans les galeries du Trocadéro»33. En dehors du palais, la fonction dévolue aux œuvres anciennes semble plus tranchée. Les présentations d'antiques assurées par certaines nations dans leurs pavillons respectifs n'ont d'autre objectif que celui de souligner, par contraste ou continuité, la grandeur industrielle du pays. La Grèce, qui confie son exposition à une Commission pour l'Encouragement de l'industrie

${ }^{26}$ Archives nationales de France F/12/3295 : Commissariat général/Services de l’État/Direction de la presse/ministère de l'intérieur. Mai-Juillet 1878.

${ }^{27}$ Archéologue et anthropologue français, membre de la commission d'initiative de l'exposition anthropologique installée dans le pavillon du quai de Billy en 1878 .

28 DE MORTILLET, Gabriel, «Exposition universelle de 1878. Palais du Trocadéro» in La Nature, 226, 29 septembre 1877, p. 274.

29 DELORME, René, in L'art et l'industrie de tous les peuples à l'Exposition Universelle de 1878. Description illustrée des merveilles du Champ de Mars et du Trocadéro par les écrivains spéciaux les plus autorisés, cit., p. 508.

$3^{30}$ Voir également «Souvenirs de l'exposition universelle de 1878», in Le Magasin Pittoresque, 46 , décembre 1878 , p. 386 : «On trouve, en visitant ces galeries, les éléments d'une histoire de l'art, et aussi, dans une certaine mesure, d'une histoire de l'industrie ; car, dans notre pays plus peut-être que dans tout autre, l'art et l'industrie se touchent par bien des points».

${ }^{31}$ L'art et l'industrie de tous les peuples à l'Exposition Universelle de 1878, cit., p. 508.

$3^{2}$ Voir par exemple BONNAFFÉ, Edmond, «Au Trocadéro. Causerie» in GONSE, Louis, L'art ancien au Trocadéro, Paris, A. Quantin, 1879, p. 18.

33 Archives nationales de France F/12/3254: Section de l'art ancien, organisation. p. 3-4. 
nationale ${ }^{34}$, affiche ce parti-pris dans l'intitulé de son comité. Ainsi, Raymond Isay se trompe en confinant l'Exposition universelle du XIXe siècle à une «machine à explorer le passé»dont «on imaginait guère qu'elle pût être [...] orientée vers le présent et l'avenir»35. Tout au contraire, la valeur industrielle de ce passé ne cesse d'être répétée à l'Exposition de 1878 pour réhabiliter l'intérêt présent de ces œuvres.

L'ambiguïté du rôle attribué aux antiques au Trocadéro reflète la cohabitation d'intérêts divers à l'exposition de l'art ancien. Garants de la manifestation, les collectionneurs profitent de l'événement pour donner à voir le témoignage de leur réussite. De leur côté, les commissions nationales cherchent à valoriser l'ancienneté de leur industrie : dans un contexte de rivalité politique internationale, la richesse d'un pays se mesure d'avantage par son progrès technique qu'à son concours archéologique.

\subsection{Le Laurion ${ }^{36}$ ou la continuité d'une pratique millénaire ${ }^{37}$}

Le cas des antiquités du Laurion nous permet d'étudier concrètement l'instrumentalisation d'objets archéologiques à des fins commerciales. Situé dans la région grecque de l'Attique, le massif minier du Laurion et ses gisements de plomb matérialisent une continuité dans la pratique minière de l'Antiquité jusqu'au XIX ${ }^{\mathrm{e}}$ siècle. "L'antiquité reculée de leur exploitation est un fait connu de tous, et personne n'essaye de dire depuis quelle époque elles sont ouvertes» écrit Xénophon en 355 avant J-C. $3^{8}$ En effet, on fait aujourd'hui remonter à l'âge de bronze l'exploitation de ces carrières. Redécouvertes dans les années 1860, les mines font l'objet d'un contentieux franco-grec en 1871. Si l' «affaire du Laurion» se solde par la dissolution de la compagnie des Métallurgies du Laurion, un nouvel exploitant français lui succède dès 1877, la Compagnie Française des Mines du Laurion. Sous l'égide de Jean-Baptiste Serpieri, l'entreprise s'affirme rapidement comme la première société minière du Laurion.

\footnotetext{
34 Lettre du commissaire N.I Delyenni à G. Berger le 15 septembre 1877. Archives nationales de France F/12/3496 : Participation des puissances étrangères. Grèce.

35 ISAY, Raymond, «L'exposition de 1878», in Revue des Deux Mondes, 38, 15 avril 1937, p. 918. ${ }^{36}$ Le terme grec classique de Laurion a été préféré à celui de Laurium, employé par A. Cordella dans ses ouvrages.

37 Je remercie Anaïs Boucher, conservatrice au Musée des Antiquités Nationales de SaintGermain-en-Laye, pour sa disponibilité et pour tout l'intérêt qu'elle a témoigné à mes recherches sur le Laurion.

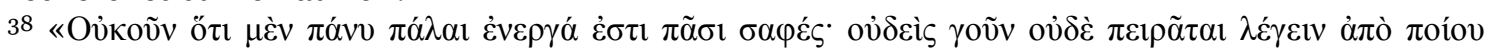

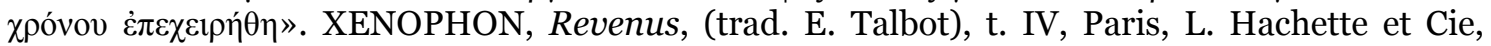
1859.
} 
La présence à l'Exposition universelle de 1878 d'objets antiques du Laurion est attestée par le aux Industries extractives, et d'une «collection d'objets antiques de l'art métallurgique, trouvée au Laurium» à l'exposition historique de l'art ancien. Toutefois, il est difficile d'établir avec précision la nature de ces objets. Dans l'ouvrage édité à l'occasion de l'Exposition de 1878, André Cordella reste allusif sur ce point, affirmant seulement que «des modèles de ces anciennes laveries, ainsi que d'un broyeur, sont faits par nous pour l'Exposition de Paris»39. L'auteur renvoie au plan d'une laverie antique, proposé dans une parution précédente ${ }^{40}$. L'inventaire de la rétrospective de l'art ancien au Trocadéro ${ }^{41}$ n'apporte aucune précision sur le contenu de ce dépôt puisqu'aucune mention n'y figure. De même, le nom de Cordella n'apparaît pas dans le répertoire des exposants.

Dans son ouvrage de 1869, Le Laurium, André Cordella énumère les «objets d'antiquités les plus intéressants »trouvés dans cette région. Cette liste offre un cadre général à l'identification des objets présentés à l'Exposition universelle. L'exploitation des carrières anciennes et les fouilles pratiquées en amont de constructions ont mis au jour des monnaies, le plus souvent découvertes dans des tombes, et des fragments de terre-cuites, céramiques et moules à lampes. Plusieurs stèles en marbre gravées ont également été récoltées. Les restes architecturaux d'un temple et d'un monument honorifique complètent cet ensemble, auquel s'ajoutent les restes des outils et procédés d'exploitation antiques. C'est à l'intérieur de ce corpus que l'on doit définir la présence archéologique de la Compagnie Française à l'Exposition. Il est tentant de voir dans les objets antiques du Laurion entrés en 1879 au Musée des Antiquités Nationales ${ }^{42}$ un échantillon du dépôt d'André Cordella à l'Exposition un an plus tôt. Mais seule la proximité chronologique entre l'Exposition et l'intégration des objets aux collections françaises légitime ce rapprochement. L'absence presque totale de documentation sur cet ensemble aux archives du Musée des Antiquités Nationales de Saint-Germain-enLaye impose de considérer cette relation avec prudence ${ }^{43}$.

39 CORDELLA, André, La Grèce sous le rapport géologique et minéralogique: à la demande de la commission centrale de la Grèce pour l'Exposition universelle de 1878, Paris, Impr. de A. Parent, 1878, p. 111.

${ }^{40}$ CORDELLA, André, Le Laurium, Marseille, impr. de Cayer, 1871, p. 95, fig. 20.

${ }^{41}$ Voir Archives nationales de France F/12/3555 et F/12/3556: Exposition historique de l'art ancien, inventaire général.

${ }^{42}$ Suite au don de l'Ingénieur des mines Alfred Huet. Les objets sont actuellement présentés au sein de la galerie d'archéologie comparée du Musée des Antiquités Nationales de SaintGermain-en-Laye.

43 Une étude de ces objets est actuellement préparée par Anaïs Boucher, conservatrice au Musée des Antiquités Nationales. 
Sans particulariser ces expôts, interrogeons-nous sur leur place, et, plus généralement, sur la considération de l'Antiquité dans la présentation de la Compagnie Française des Mines du Laurion au Palais du Champ de Mars. Chargé par la commission grecque d'entreprendre un compte rendu de l'état des industries extractives du pays en 1878, André Cordella insiste sur l'ancienneté de la pratique extractive au Laurion, donnant ainsi une certaine noblesse au site 44 :

\begin{abstract}
Dans les parties faibles de ces antiques travaux, le toit est soutenu par des piliers de maçonnerie en pierres sèches, si bien conservées encore, qu'ils semblent être construits depuis quelques années seulement.

On y remarque très bien les sentiers par où passaient les esclaves chargés de minerais ; les petites colonnes qui leur servaient de guides, les niches où étaient déposées les lampes destinées à éclairer leur marche, et des cruches pour conserver leur eau45.
\end{abstract}

Après l'«Affaire du Laurion», l'insistance sur ce passé antique traduit une forme de continuité entre l'activité des Athéniens du Ve siècle avant J-C. et celle des mineurs de l'entreprise française, légitimant ainsi l'exploitation des sols grecs par la Compagnie. Le remploi des puits anciens pour faciliter l'aérage des galeries modernes donne corps à une pérennité qui se veut également matérielle. «Lorsque l’on enlève les déblais [...], on est tenté de croire que l'exploitation des mines est suspendue depuis quelques années seulement, et qu'elle ne demande qu'à être reprise» ${ }^{46}$ justifie André Cordella. D’une façon générale, les sociétés minières implantées au Laurion se rattachent fréquemment au passé grec, à l'image des compagnies Périclès et Nikias. De la même façon, l'ancienneté des carrières devient un élément de publicité pour la Compagnie Française lors des concours industriels. Des objets archéologiques ont ainsi été présentés par la société à Athènes lors des expositions Olympiennes de 1870 et $1875^{47}$.

\footnotetext{
44 Cette position est plus marquée dans CORDELLA, André, Le Laurium, cit., André Cordella y consacre une partie aux «antiquités du Laurium», pp. 31 à 40, et un chapitre à «l'Industrie minière et métallurgique des anciens», pp. 79 à 109.

45 CORDELLA, André, La Grèce sous le rapport géologique et minéralogique, cit., 1878, p. 107.

${ }^{46}$ Ibidem, pp. 110-111.

47 Olympiada de la deuxième et de la troisième période. Pour chacune de ces deux expositions, André Cordella se charge d'une publication décrivant les dépôts de la société. Voir pour 1870:

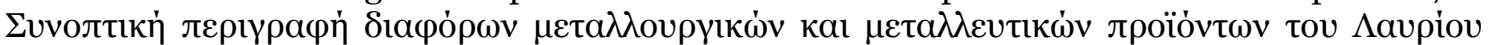

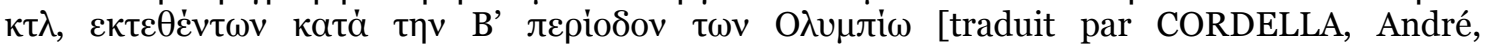
Description des produits des mines et des usines du Laurium e.c.t. exposés à la seconde période

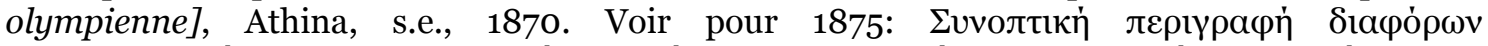

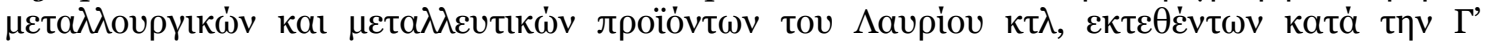
$\pi \varepsilon \rho i o \delta o v ~ \tau \omega v ~ O \lambda v \mu \pi i \omega v$ [Description des produits des mines et des usines du Laurium et d'Oropos exposés à la troisième période olympienne], Athina, s.e., 1875.
} 
Ingénierie et matériel antiques 48 accompagnent la démonstration des procédés modernes et autres innovation techniques, de sorte à garantir la permanence de la pratique ouvrière au fil des siècles.

Ainsi, le dépôt d'André Cordella au Trocadéro répond aux ambitions officielles précédemment énoncées au sujet de l'exposition historique de l'art ancien. Rattachés à l'histoire de l'art par leur antiquité, et fondamentalement associés au monde industriel par leur fabrication artisanale et leur contexte d'utilisation, ces objets accomplissent la jonction des deux univers artistique et industriel dont Gabriel de Mortillet anticipe la fusion en 1877.

${ }^{48}$ La description de ce matériel est fournie par CORDELLA, André, Le Laurium, cit., 1871, p. 87: «Les outils des mineurs étaient très-simples, et d'après les traces qu'ils ont laissés dans les roches, il est facile de les ranger en trois catégories. Un pic à pointe conique employé dans les roches dures, un autre à pointes pyramidales pour l'exploitation des filons et des roches tendres; enfin, les derniers étaient des coins de dimensions diverses; il est probable qu'ils avaient aussi des marteaux et des masses ainsi que des leviers». 


\section{Conclusion}

On appelle volontiers les arts et les lettres les fruits de la paix, pacis fructus. Il semble au contraire qu'ils naissent et qu'ils se développent dans les fureurs de la guerre [...]. C'est après Salamine et Platées que s'épanouit le génie hellénique; c'est après les longues guerres de César que chantent les poètes latins49.

Henry Houssaye souligne ici ce qui fait l'originalité de l'Exposition universelle de 1878. Première exposition républicaine, l'événement signe le redressement de la France après le désastre de la guerre franco-prussienne et de la Commune. Indirectement, la métaphore de l'auteur rend compte des liens étroits qui unissent l'événement à l'Antiquité. Ce rapport est matérialisé par la présence de dépôts archéologiques à l'Exposition. Perçu pour son intérêt documentaire par les spécialistes, ce legs archéologique est bien plus souvent support aux idéologies contemporaines chez les commentateurs et le grand public. Les objets antiques accompagnent notamment les revendications laïques et nationalistes d'une société en mutation. Au regard du contexte contemporain, le lien entre passé et présent semble s'inscrire en toile de fond de l'Exposition de 1878. La confrontation des productions anciennes aux produits de l'industrie fait écho au dépassement de l'ancien Empire, supplanté par l'avènement de la République. «L'Exposition, dans tous les domaines, illustre cette heure de transition: entre l'expérience qui commence et le passé qui s'évanouit, elle représente un compromis, mais aussi une sorte de synthèse, d'harmonie et de fusion»écrit Raymond Isay50. Les dimensions de progrès et de dépassement sont prégnantes en 1878. À la distance temporelle qui sépare les objets antiques des créations du monde moderne correspond la distance idéologique entre les deux régimes. Pour autant, des liens se mesurent entre legs ancien et développements modernes.

49 HOUSSAYE, Henri, «Voyage autour du monde à l'exposition universelle II: l'Europe méridionale, l'Europe du Nord, l'Europe centrale, la France» in Revue des deux mondes, 28, 15 juillet 1878, p. 802.

50 ISAY, Raymond, op.cit., p. 923. 


\section{* L'auteur}

Bastien Noël est diplômé en histoire de l'art et muséologie à l'Ecole du Louvre (Paris); il a rédigé en 2011 un mémoire d'étude portant sur «Les antiques à l'Exposition universelle de 1878, à Paris», sous la direction d'Edouard Vasseur. En 2012, dans le cadre de la journée d'étude «Les expositions universelles - nouveaux chantiers de recherche», il présente une partie de ses travaux à travers une communication sur «Le quotidien antique à l'Exposition universelle de 1878» (en juin 2014).

URL: < http://www.studistorici.com/progett/autori/\#Noël >

\section{Per citare questo articolo:}

NOËL, Bastien, «Exposition universelle de 1878. L'archéologie au service des contestations contemporaines», Diacronie. Studi di Storia Contemporanea : Le esposizioni: propaganda e costruzione identitaria, 29/6/2014, URL: < http://www.studistorici.com/2014/6/29/noel_numero_18/ >

Diacronie Studi di Storia Contemporanea $\}$ www.diacronie.it

Risorsa digitale indipendente a carattere storiografico. Uscita trimestrale. redazione.diacronie@hotmail.it

Comitato di redazione: Jacopo Bassi - Luca Bufarale - Elisa Grandi - Deborah Paci - Fausto Pietrancosta - Matteo Tomasoni - Luca Zuccolo 